Pacific Journal of Mathematics

ENTIRE SOLUTIONS OF LINEAR ELLIPTIC EQUATIONS 


\title{
ENTIRE SOLUTIONS OF LINEAR ELLIPTIC EQUATIONS WITH LAPLACIAN PRINCIPAL PART
}

\author{
Gerald N. Hile
}

Consider the equations in $R^{n}, n \geqq 2$,

$$
\begin{aligned}
& \Delta \varphi=f+b \cdot \nabla \varphi \\
& \Delta \varphi=b \cdot \nabla \varphi
\end{aligned}
$$

where $f$ and $b$ are locally Hölder continuous, and as $|x| \rightarrow \infty$, $f(x)=O\left(|x|^{-\tau}\right), b(x)=O\left(|x|^{-\sigma}\right), \sigma, \tau>1$. It is shown that if $0 \leqq \rho<\sigma-1$, there is a one-to-one correspondence between entire $C^{2}$ solutions of $(*)$ whose gradients grow no faster than $O\left(|x|^{\rho}\right)$, and harmonic polynomials with gradients of the same growth. For (I) therefore solutions whose gradients grow no faster than $O\left(|x|^{\rho}\right)$ form a finite dimensional vector space. These results for (I) give analogues to the concept of "generating pairs" in pseudo-analytic function theory.

1. Introduction. In the case $n=2$, (I) takes the form

$$
\varphi_{x x}+\varphi_{y y}=b_{1} \varphi_{x}+b_{2} \varphi_{y}
$$

If we make the identifications $w=\varphi_{x}-i \varphi_{y}, \quad A=\left(b_{1}+i b_{2}\right) / 4, \quad B=$ $\left(b_{1}-i b_{2}\right) / 4$, then $w$ satisfies the complex equation

$$
\frac{\partial w}{\partial \bar{z}}=A w+B \bar{w}
$$

where

$$
\frac{\partial}{\partial \bar{z}}=\frac{1}{2}\left(\frac{\partial}{\partial x}+i \frac{\partial}{\partial y}\right)
$$

Thus (1.1) can be studied alternatively by considering the complex equation (1.2), the solutions of which are known as "pseudo-analytic functions," and for which an extensive theory has been developed (see for example the treatments of Bers and Vekua in [1] and [8]). In particular it is known that entire and bounded solutions of (1.2) form a two-dimensional real vector space, and a basis for this vector space is called a "generating pair." In dimensions higher than two the reduction of (I) to a first order complex equation. is no longer 
feasible. However in the presentation here several features of the two-dimensional treatment are retained, with the main essential step being the establishment of a relation between solutions of $(*)$ and solutions of a prescribed integral equation.

Entire solutions of linear second order elliptic equations are discussed by D. Gilbarg and J. Serrin in [4], and by A. Friedman in [3]. These results are stated in terms of the behavior of the solutions themselves, whereas results here describe the behavior of the gradients of solutions. Gilbarg and Serrin give a Liouville's theorem for elliptic equations with principal part much more general than the Laplacian, and with the coefficients of the first order terms behaving like $O\left(|x|^{-1}\right)$ at infinity. The proofs of one or two of the results here, particularly Theorem 8, could be shortened slightly by quoting results from [4]. Otherwise there does not appear to be much overlap between that paper and this one. Friedman in [3] gives conditions under which a rather general elliptic equation is known to have an entire and bounded solution. Connections of his results with results here are briefly described in section 3 .

J. Serrin in [7] has developed Liouville-type theorems for nonlinear equations of the form $\Delta \varphi=f(x, \varphi, \nabla \varphi)$. His results are stated in terms of the behavior of both the solution and the gradient of the solution. He also gives a Liouville theorem for a special case of (I) where $b$ is no longer required to vanish at infinity but other restrictive conditions are added.

Another generalization of the concept of generating pairs is given in [5] by the present author with R. P. Gilbert. In this work an analogue of pseudo-analytic function theory is developed for certain elliptic systems of first order equations in the plane.

We briefly describe some function spaces. All functions are presumed defined everywhere in $R^{n}$. Let $f$ map $R^{n}$ into $R$. We say $f \in C^{m}$ if all derivatives of $f$ up to order $m$ are continuous. We say $f \in H$, or $f$ is locally Hölder continuous, if for each compact set $K$ in $R^{n}$ there exist positive constants $M$ and $\beta, 0<\beta \leqq 1$, such that for $x$, $y \in K$,

$$
|f(x)-f(y)| \leqq M|x-y|^{\beta}
$$

We say $f \in H_{\alpha}$ if we may choose $\beta=\alpha$ for each compact set $K$. Let $\rho$ be a real number. We say $f \in B_{\rho}$ if $f$ is continuous and $f(x)=$ $O\left(|x|^{\rho}\right)$ as $|x| \rightarrow \infty$. The space $B_{\rho}$ is a Banach space under the norm

$$
\|f\|_{\rho}=\sup _{x \in R^{n}} \frac{|f(x)|}{(1+|x|)^{\rho}}
$$


The space $B_{0}$ consists of all bounded continuous functions in $R^{n}$.

If $u$ is a vector valued function from $R^{n}$ into $R^{n}$, we say $u$ lies in one of the above spaces provided that each real component of $u$ is in that space. We distinguish separately the space $B_{\rho}(n)$, consisting of real $n$-vector valued functions $u=\left(u_{1}, \cdots, u_{n}\right)$ with each component in $B_{\rho}$. The norm in this space is described by (1.3) with $f$ replaced by $u$, and where we interpret $|u(x)|$ as the ordinary Euclidean norm of the vector $u(x)$.

2. Properties of operators. In this section we develop properties of operators to be used later. We list as lemmas several elementary consequences of known results involving potentials in $R^{n}$, $n \geqq 2$. We denote the gradient operator by $\nabla=\left(\partial_{1}, \cdots, \partial_{n}\right)$, and the Laplacian by $\Delta=\partial_{11}+\cdots+\partial_{n n}$. The surface area of the unit sphere in $R^{n}$ is $\omega_{n}$, and $d y=d y_{1} \cdots d y_{n}$ represents $n$-dimensional Lebesgue measure. If $g$ is a real valued function in $R^{n}$, we define formally the potential $S g$ by

$$
\begin{aligned}
& (S g)(x)=\frac{1}{(2-n) \omega_{n}} \int_{R^{n}}|x-y|^{2-n} g(y) d y \quad \text { if } \quad n \geqq 3 \\
& =\frac{1}{2 \pi} \int_{R^{2}} \log |x-y| g(y) d y \quad \text { if } \quad n=2 .
\end{aligned}
$$

We denote by $T g$ the formal gradient of $S g$,

$$
(T g)(x)=\frac{1}{\omega_{n}} \int_{R^{n}} \frac{x-y}{|x-y|^{n}} g(y) d y \quad \text { if } \quad n \geqq 2 .
$$

If $u$ is a function from $R^{n}$ into $R^{n}, u=\left(u_{1}, \cdots, u_{n}\right)$, we define formally

$$
(R u)(x)=\frac{1}{\omega_{n}} \int_{R^{n}} \frac{(x-y) \cdot u(y)}{|x-y|^{n}} d y \quad \text { if } \quad n \geqq 2,
$$

where "." is the ordinary dot product for vectors.

Lemma 1. If $g \in H \cap B_{-\sigma}$ where $\sigma>1$, then $T g \in C^{1}$, and in $R^{n}$ we have

$$
\nabla \cdot T g=g
$$

Moreover, $T g$ is the gradient of some $C^{2}$ function in $R^{n}$.

Proof. The condition $\sigma>1$ guarantees that the integral $\mathrm{Tg}$ converges. For any fixed $r, r>0$, we write $T=T_{r}+T_{r}^{\prime}$, where 


$$
\begin{aligned}
& \left(T_{r} g\right)(x)=\frac{1}{\omega_{n}} \int_{|y| \leqq r} \frac{x-y}{|x-y|^{n}} g(y) d y \\
& \left(T_{r}^{\prime} g\right)(x)=\frac{1}{\omega_{n}} \int_{|y| \geqq r} \frac{x-y}{|x-y|^{n}} g(y) d y .
\end{aligned}
$$

Similar decompositions hold for $S$ and $R$. By well known results for bounded domains (see for example [2], Ch. IV), $S_{r} g$ and $T_{r} g$ have continuous derivatives of orders two and one, respectively, in the region $|x|<r$, and satisfy there

$$
\begin{gathered}
\nabla\left(S_{r} g\right)=T_{r} g \\
\nabla \cdot\left(T_{r} g\right)=\Delta\left(S_{r} g\right)=g .
\end{gathered}
$$

Moreover for $|x|<r$ we may differentiate under the integral and obtain $\nabla \cdot\left(T_{r}^{\prime} g\right)=0$, which together with (2.6) gives (2.4). To see that $T g$ is the gradient of a real valued $C^{2}$ function in $R^{n}$, we write, for $r>0, u=T_{r} g+T_{r}^{\prime} g=T g$. Since $u \in C^{1}$, it is sufficient to show $u$ satisfies the system

$$
\frac{\partial u_{l}}{\partial x_{j}}=\frac{\partial u_{I}}{\partial x_{i}}, \quad i, j=1, \cdots, n ; i \neq j
$$

However $T_{r} g$ satisfies equations of this form in the region $|x|<r$, since $T_{r} g=\nabla\left(S_{r} g\right)$ in this region, and $T_{r}^{\prime} g$ satisfies equations of this form in the same region because we may differentiate under the integral.

The next two theorems are stated here and their proofs, being mainly calculational in nature, are delayed until the last section.

Theorem 2. If $g \in B_{-\sigma}$ where $1<\sigma<n$, then $T g \in H_{\alpha} \cap B_{1-\sigma}(n)$ for any $\alpha$ in the range $0<\alpha<1$, and

$$
\|T g\|_{1-\sigma} \leqq M(n, \sigma)\|g\|_{-\sigma} .
$$

If $u \in B_{-\sigma}(n)$ where $1<\sigma<n$, then $R u \in H_{\alpha} \cap B_{1-\sigma}$ for $0<\alpha<1$, and

$$
\|R u\|_{1-\sigma} \leqq M(n, \sigma)\|u\|_{-\sigma}
$$

$(M(n, \sigma)$ denotes a positive constant depending only on $n$ and $\sigma$.

TheOREM 3. If $b \in B_{-\sigma}(n)$ where $\sigma>1$, then the operator $P$ defined by $P u=T(b \cdot u)$ is compact in the space $B_{\rho}(n)$ for $1-n<\rho<$ $\sigma-1$. 
LEMMA 4. Let $\varphi$ be a real valued function in $R^{n}, \varphi \in C^{2}$, $\Delta \varphi \in H \cap B_{-\sigma}$ where $\sigma>1$. Then there exists an entire harmonic function $h$, unique up to an additive constant, such than in $R^{n}$

$$
\nabla \varphi=\nabla h+T(\Delta \varphi)
$$

Proof. By Lemma 1, $T(\Delta \varphi)=\nabla \psi$ for some function $\psi$ in $C^{2}$, and

$$
\nabla \cdot(\nabla \varphi-\nabla \psi)=\Delta \varphi-\nabla \cdot T(\Delta \varphi)=\Delta \varphi-\Delta \varphi=0
$$

Thus $\varphi-\psi=h$ for some entire harmonic function $h$, and $\nabla \varphi=$ $\nabla h+\nabla \psi=\nabla h+T(\Delta \varphi)$. If there were two functions with the properties of $h$, the gradient of their difference would be zero.

Lemma 5. If $\varphi \in C^{1}, \nabla \varphi \in B_{-\tau}(n)$ where $\tau>1$, then

$$
\varphi=c+R(\nabla \varphi)
$$

for some real number $c$.

Proof. We use integration by parts to derive, for $|x|<r, \epsilon>0$,

$$
\begin{aligned}
& \frac{1}{\omega_{n}} \int_{|y-x| \geqq \epsilon,|y| \leqq r} \frac{(x-y) \cdot(\nabla \varphi)(y)}{|x-y|^{n}} \\
& \quad=\frac{1}{\omega_{n}}\left[\int_{|y|=r}+\int_{|y-x|=\epsilon}\right] \frac{(x-y) \cdot \nu(y)}{|x-y|^{n}} \varphi(y) d s
\end{aligned}
$$

( $d s=$ surface measure, $\nu=$ unit normal pointing outward). On the surface $|y-x|=\epsilon, \nu(y)=-(y-x) /|y-x|$, and after letting $\epsilon$ approach zero we obtain

$$
R_{r}(\nabla \varphi)(x)=\frac{1}{\omega_{n}} \int_{|y|=r} \frac{(x-y) \cdot y}{|x-y|{ }^{n} r} \varphi(y) d s+\varphi(x)
$$

The integral on the right side of (2.11) is harmonic in the region $|x|<r$. As $r$ approaches infinity, $R_{r}(\nabla \varphi)$ approaches $R(\nabla \varphi)$, and it is easily shown that convergence is uniform on compact subsets of $R^{n}$. Thus the harmonic functions defined by the integrals in (2.11) converge uniformly on compact subsets of $R^{n}$ to some harmonic function $h$, as $r$ approaches infinity, and we have the equation

$$
\varphi=h+R(\nabla \varphi)
$$


By Theorem 2, $R(\nabla \varphi)$ vanishes at infinity. By applying elementary estimates to the identity

$$
\varphi(x)=\varphi(0)+\int_{0}^{1}(\nabla \varphi)(t x) \cdot x d t
$$

(using $\nabla \varphi \in B_{-\tau}(n)$ ), we see that $\varphi$ is bounded and hence $h$ is constant.

3. The nonhomogeneous equation. We consider the two equations in $R^{n}$,

$$
\begin{aligned}
\Delta \varphi & =f+b \cdot \nabla \varphi \\
u & =\nabla q+T f+T(b \cdot u)
\end{aligned}
$$

where $q$ represents a harmonic polynomial. We formulate conditions under which solutions of $(* *)$ correspond to gradients of solutions of $(*)$.

TheOREM 6. Let $b \in H \cap B_{-\sigma}(n), f \in H \cap B_{-\tau}$, where $\sigma, \tau>1$. If $u \in B_{\rho}(n)$ where $0 \leqq \rho<\sigma-1$, then $u$ is the gradient of some $C^{2}$ solution $\varphi$ of $(*)$ if and only if $u$ satisfies $(* *)$ for some harmonic polynomial $q$ with (degree $q$ ) $\rho+1$.

Proof. If $\varphi \in C^{2}$ and satisfies (*) with $\nabla \varphi \in B_{\rho}(n)$, then $\Delta \varphi \in$ $H \cap B_{-\xi}$ where $\xi=\min (\sigma-\rho, \tau)$. By Lemma 4 , there exists an entire harmonic function $h$ such that

$$
\nabla \varphi=\nabla h+T(\Delta \varphi)=\nabla h+T f+T(b \cdot \nabla \varphi) .
$$

Since $T(\Delta \varphi)$ vanishes at infinity (Theorem 2), we have $\nabla h \in B_{\rho}(n)$, and $h$ is a polynomial of degree no larger than $\rho+1$.

Conversely suppose $u \in B_{\rho}(n)$ and satisfies $(* *)$ for some harmonic polynomial $q$, (degree $q) \leqq \rho+1$. Then $(f+b \cdot u) \in B_{-\xi}, \xi=$ $\min (\sigma-\rho, \tau)$. By Theorem 2, $T(f+b \cdot u) \in H$ and hence $u \in H$ (by $(* *)), \quad b \cdot u \in H$. By Lemma 1, $T(f+b \cdot u) \in C^{1}, \nabla \cdot T(f+b \cdot u)=$ $f+b \cdot u$, and $T(f+b \cdot u)=\nabla \psi$ for some function $\psi$ in $C^{2}$. Thus $u=\nabla q+\nabla \psi=\nabla \varphi$ where $\varphi=q+\psi$. Moreover, $\Delta \varphi=\Delta(q+\psi)=\Delta \psi=$ $\nabla \cdot T(f+b \cdot u)=f+b \cdot u=f+b \cdot \nabla \varphi$.

LEMMA 7. Let $b \in H \cap B_{-\sigma}(n), f \in H \cap B_{-\tau}$, where $\sigma>1,1<$ $\tau<n$. If $\varphi$ is a $C^{2}$ solution of $(*)$ whose gradient vanishes at infinity, then $\nabla \varphi \in B_{1-\tau}(n)$. 
Proof. Since $b \cdot \nabla \varphi \in B_{-\sigma}, f \in B_{-\tau}$, Theorem 2 implies $T f+$ $T(b \cdot \nabla \varphi)$ vanishes at infinity, and in $(* *)$ (with $u=\nabla \varphi$ ) we have $\nabla q=0$. Thus

$$
\nabla \varphi=T(f+b \cdot \nabla \varphi) .
$$

If $\sigma \geqq \tau$, then $f+b \cdot \nabla \varphi \in B_{-\tau}$ and by Theorem $2, \nabla \varphi \in B_{1-\tau}(n)$. If $\sigma<\tau$, we have $f+b \cdot \nabla \varphi \in B_{-\sigma}$ and $\nabla \varphi \in B_{1-\sigma}(n)$, which gives $b \cdot \nabla \varphi \in B_{1-2 \sigma}$. If $2 \sigma-1 \geqq \tau$, then as before we have $f+b \cdot \nabla \varphi \in B_{-\tau}$, $\nabla \varphi \in B_{1-\tau}(n)$. If $2 \sigma-1<\tau$, then $f+b \cdot \nabla \varphi \in B_{1-2 \sigma}, \nabla \varphi \in B_{2-2 \sigma}(n)$. Continuing in this manner, we obtain $b \cdot \nabla \varphi \in B_{-\sigma-i(\sigma-1)}$ for succeeding integers $i$, until $\sigma+i(\sigma-1) \geqq \tau$, which then gives $\nabla \varphi \in B_{1-\tau}(n)$.

Remark. One can show that $\nabla \varphi \in B_{1-n}(n)$ if $\tau>n$, and if $\tau=n$, $\nabla \varphi(x)=O\left(|x|^{1-n} \log |x|\right)$. These results rely on an extension of Theorem 2 to the case $\sigma \geqq n$, whose proof in the interests of brevity is not included.

Finally, we discuss solvability of $(*)$ :

THEOREM 8. Let $b \in H \cap B_{-\sigma}(n), f \in H \cap B_{-\tau}$, where $\sigma, \tau>$ 1. If $\varphi$ is a $C^{2}$ solution of $(*)$ with $\nabla \varphi \in B_{\rho}(n)$ where $0 \leqq \rho<\sigma-1$, then there exists a harmonic polynomial $q$, with (degree $\nabla q) \leqq \rho$, such that $\nabla \varphi-\nabla q$ vanishes at infinity. Conversely, for any given harmonic polynomial $q$ with (degree $\nabla q)=m, m<\sigma-1$, there exists a $C^{2}$ solution $\varphi$ of $(*)$, uniquely determined up to an additive constant, such that $\nabla \varphi \in B_{m}(n)$ and $\nabla \varphi-\nabla q$ vanishes at infinity.

Proof. The first half of the theorem follows from Theorem 6 . From $(* *)$ we have the representation

$$
\nabla \varphi=\nabla q+T(f+b \cdot \nabla \varphi)
$$

for some harmonic polynomial $q$, (degree $q$ ) $\rho+1$. Since $f \in B_{-\tau}$, $(b \cdot \nabla \varphi) \in B_{\rho-\sigma}$, the quantity $T(f+b \cdot \nabla \varphi)$ vanishes at infinity. For the "conversely" part, it is sufficient to show the equation $(* *)$ has a unique solution in the space $B_{m}(n)$ for any given harmonic polynomial $q$ with $\nabla q \in B_{m}(n), 0 \leqq m<\sigma-1$. Since $T f \in B_{1-\tau}(n)$, and $B_{1-\tau}(n) \subset$ $B_{m}(n)$ for $m \geqq 0$, and the operator $P$ given by $P u=T(b \cdot u)$ is compact in the space $B_{m}(n)$, it is sufficient to show that the only solution to the homogeneous form of $(* *)$ is the zero solution. Suppose then that $u \in B_{m}(n)$, with $0 \leqq m<\sigma-1$, and we have $u=T(b \cdot u)$. Since $b \cdot u \in B_{-(\sigma-m)}$ and $\sigma-m>1, T(b \cdot u)$ vanishes at infinity. For the special case $n=2$, it is proved in [1], pp. 59-64, making use of the similarity principle for pseudo-analytic 
functions, that $u$ is identically zero. We assume therefore that $n \geqq 3$. We let $u=\nabla \psi$, where $\psi$ is a $C^{2}$ solution of $\Delta \psi=b \cdot \nabla \psi$ (Theorem 6). Applying Lemma 7 with $f=0,2<\tau<n$, we conclude $\nabla \psi \in B_{-\xi}$ for some $\xi, \xi>1$. By Lemma $5, \psi=c+R(\nabla \psi)$ for some real constant $c$. Since $R(\nabla \psi)$ vanishes at infinity $\psi$ has the limit $c$ at infinity. By the maximum principle the function $\psi-c$ is identically zero, and hence $u=\nabla \psi=0$.

REMARK. Under the conditions of Theorem 8, there exists a unique (up to an additive constant) $C^{2}$ solution $\varphi$ of $(*)$ whose gradient vanishes at infinity, occurring in the case $\nabla q=0$. In [3] it is shown that under the condition of Theorem 8 , except with $\tau>2$, there exists a unique solution of $(*)$ vanishing at infinity for $n \geqq 3$. (In fact, this result is proved for an elliptic equation with principal part much more general than the Laplacian.) Lemma 7 shows that furthermore the gradient of this unique solution vanishes at infinity. Indeed, Lemma 7 guarantees in the case $\tau>2, n \geqq 3$ that $\nabla \varphi \in B_{-\rho}(n)$ for some $\rho, \rho>1$. By Lemma 5, $\varphi$ has a limit at infinity and thus $\varphi$ differs by a constant from the unique solution vanishing at infinity. (For the case $n=2, \rho>2$, the remark after Lemma 7 guarantees existence of a solution with $\nabla \varphi \in B_{-1}(n)$, which with the help of (2.12) leads to $\varphi(x)=O(\log |x|)$. In [3] the existence of a solution with this growth at infinity is also proved.)

4. The homogeneous equation. We assume throughout this section that $b \in H \cap B_{-\sigma}(n)$ where $\sigma>1$, and consider the equation in $R^{n}$,

$$
\Delta \varphi=b \cdot \nabla \varphi
$$

The next theorems are immediate consequences of Theorems 2, 6, and 8 and the linearity of the equation.

THEOREM 9. If $0 \leqq \rho<\sigma-1$, the collection of gradients of $C^{2}$ solutions of (I) whose gradients are in $B_{\rho}(n)$ is a finite dimensional real vector space. This vector space is isomorphic to the space of gradients of harmonic polynomials $q$ in $B_{\rho+1}(n)$, and a one-to-one correspondence between the two spaces is determined by the relation $\nabla \varphi-\nabla q=O$ $\left(|x|^{-\tau}\right)$ for some $\tau, \tau>0$. (If $\sigma-\rho<n$, then $\tau=\sigma-\rho-1$.)

THEOREM 10. The collection of gradients of $C^{2}$ solutions of (I) whose gradients are bounded is an $n$-dimensional real vector space. $A$ one-to-one correspondence between this space and $R^{n}$ is determined by the relation, for $\gamma$ in $R^{n}, \nabla \varphi-\gamma=O\left(|x|^{-\tau}\right)$ for some $\tau, \tau>0$, (If $\sigma<n$, then $\tau=\sigma-1$.) 
Definition. For given $b \in H \cap B_{-\sigma}(n), \sigma>1$, and given $\rho$, $0 \leqq \rho<\sigma-1$, we define $G(b, \rho)$ as the real vector space of gradients of $C^{2}$ solutions of (I) whose gradients are in $B_{\rho}(n)$.

Definition. A generating set for $G(b, \rho)$ is a collection of elements in $G(b, \rho)$ that forms a basis for this vector space.

We note that $G(b, 0)$ is the space of bounded gradients described in Theorem 10. For the case $n=2$, in the language of pseudoanalytic function theory elements of this space are called generalized constants, and a generating set is called a generating pair. If we let $e_{i}$ denote the unit vector in the ith-coordinate direction in $R^{n}$, and denote by $\varphi_{1}$ a $C^{2}$ solution of (I) whose gradient has the limit $e_{\text {, }}$ at infinity, then clearly the set $\nabla \varphi_{1}, \cdots, \nabla \varphi_{n}$ is a generating set for $G(b, 0)$. In fact, if $\nabla \varphi$ has limit $\gamma=\left(\gamma_{1}, \cdots, \gamma_{n}\right)$ at infinity, where $\varphi$ is a $C^{2}$ solution of (I), then $\nabla \varphi$ has the representation $\nabla \varphi=$ $\Sigma \gamma_{i} \nabla \varphi_{i}$. Note also that since $\nabla \varphi_{i} \rightarrow e_{i}$ at infinity, there exists a neighborhood of infinity in $R^{n}$ for which the set of $n$-vectors $\left(\nabla \varphi_{1}\right)(x), \cdots,\left(\nabla \varphi_{n}\right)(x)$ is linearly independent at each point $x$ in that neighborhood. A natural question is whether the set $\left(\nabla \varphi_{1}\right)(x)$, $\cdots,\left(\nabla \varphi_{n}\right)(x)$ is linearly independent at every point $x$ in $R^{n}$. Equivalent formulations of the same problem are as follows:

(i) For fixed $x$ in $R^{n}$, is it true that $R^{n}=\{\nabla \varphi(x): \nabla \varphi \in G(b, 0)\}$ ?

(ii) If $\nabla \varphi \in G(b, 0)$ and $\nabla \varphi$ vanishes at some point, is $\nabla \varphi$ identically zero?

For the case $n=2$, the answer to these questions is affirmative, and follows as a trivial consequence of the similarity principle for pseudoanalytic functions. For $n \geqq 3$ this function theory is not available, the main hindrance being the lack of a suitable commutative algebra with which to represent points in $R^{n}$. The next theorem gives an affirmative answer to (i) and (ii) whenever the norm $\|b\|_{-\sigma}$ is sufficiently small, but for the more general case these questions remain unanswered.

THEOREM 11. If $\|b\|_{-\sigma}$ is sufficiently small (the bound depending on $n$ and $\sigma$ ), and if $\varphi$ is a $C^{2}$ solution of (I) with bounded gradient, then $\nabla \varphi$ never vanishes unless $\nabla \varphi$ is identically zero.

Proof. By choosing $\sigma$ smaller if necessary, we assume $1<\sigma<n$. We will prove the anwer to (i) is affirmative. If $\nabla \varphi \in G(b, 0)$, then $\nabla \varphi$ has the representation

$$
\nabla \varphi=\gamma+T(b \cdot \nabla \varphi)=\gamma+P(\nabla \varphi)
$$

for some constant $n$-vector $\gamma$. Let $x$ be fixed in $R^{n}$. The condition $(\nabla \varphi)(x)=y$ leads to $\gamma=y-P(\nabla \varphi)(x)$, and 


$$
\nabla \varphi=y+P(\nabla \varphi)-P(\nabla \varphi)(x)
$$

Thus it is sufficient to show that the equation $u=y+P u-P u(x)$ is solvable in $B_{0}(n)$ for any $y$ in $R^{n}$. For this purpose it is sufficient to show that the mapping $A$ given by $A u=y+P u-P u(x)$ is a contraction in the space $B_{0}(n)$. We have by Theorem 2, for $u$, $v \in B_{0}(n)$,

$$
\begin{aligned}
\|A u-A v\|_{0} & \leqq\|P u-P v\|_{0}+|P u(x)-P v(x)| \\
& \leqq 2\|P u-P v\|_{0} \leqq 2\|P(u-v)\|_{1-\sigma} \\
& \leqq 2 M(n, \sigma)\|b \cdot(u-v)\|_{-\sigma} \\
& \leqq 2 M(n, \sigma)\|b\|_{-\sigma}\|u-v\|_{0} .
\end{aligned}
$$

Thus we require $2 M(n, \sigma)\|b\|_{-_{\sigma}}<1$.

5. Calculations. In this section Theorems 2 and 3 are proved. The presentation follows somewhat that of [1] for the case of two dimensions. We designate generic constants by $M()$, where inside the parenthesis are listed the entities that determine $M$.

LEMMA 12. Let $x$ and $y$ be elements in an inner product space (either real or complex) with inner product $x \cdot y$, and $|x|^{2}=x \cdot x$. If $\nu>0,|x| \neq|y|$, then

$$
\left.|x| x\right|^{\nu}-y|y|^{\nu}\left|\leqq \frac{|x|^{\nu+1}-|y|^{\nu+1}}{|x|-|y|}\right| x-y \mid
$$

and if $x \neq 0, y \neq 0$, then

$$
\left|\frac{x}{|x|^{\nu+2}}-\frac{y}{|y|^{\nu+2}}\right| \leqq \frac{|x|^{\nu+1}-|y|^{\nu+1}}{|x|-|y|} \cdot \frac{|x-y|}{|x|^{\nu+1}|y|^{\nu+1}}:
$$

Equality holds in both cases, except when $x$ or $y$ is zero, only when $y$ is a positive multiple of $x$.

Proof. If $\nu>-1$ the right side of (5.1) is positive, and it is equivalent to show

$$
\left.|x| x\right|^{\nu}-\left.y|y|^{\nu}\right|^{2}(|x|-|y|)^{2} \leqq\left(|x|^{\nu+1}-|y|^{\nu+1}\right)^{2}|x-y|^{2} .
$$

We expand each side to obtain

$$
\begin{aligned}
& {\left[|x|^{2 \nu+2}+|y|^{2 \nu+2}-(x \cdot y+y \cdot x)|x|^{\nu}|y|^{\nu}\right]\left[|x|^{2}-2|x||y|+|y|^{2}\right]} \\
& \quad \leqq\left[|x|^{2 \nu+2}-2|x|^{\nu+1}|y|^{\nu+1}+|y|^{2 \nu+2}\right]\left[|x|^{2}-(x \cdot y+y \cdot x)+|y|^{2}\right] .
\end{aligned}
$$


After cancelling like terms, moving all terms to the right side, and factoring, we obtain the equivalent expression

$$
0 \leqq(2|x||y|-x \cdot y-y \cdot x)\left(|x|^{\nu+2}-|y|^{\nu+2}\right)\left(|x|^{\nu}-|y|^{\nu}\right) \text {. }
$$

The first factor on the right side is nonnegative, and the last two factors have the same sign if $\nu>0$. (Note that if $-1<\nu<0$, the last two factors have opposite signs, and therefore the opposite inequality holds in (5.1).) Equality holds in (5.3) if and only if the first factor on the right is zero, which happens only when $x=0, y=0$, or $y$ is a positive multiple of $x$. To prove (5.2), we apply (5.1) to the identity

$$
\left|\frac{x}{|x|^{\nu+2}}-\frac{y}{|y|^{\nu+2}}\right|=\frac{\left.|x| x\right|^{\nu}-y|y|^{\nu} \mid}{|x|^{\nu+1}|y|^{\nu+1}}
$$

which can be verified by squaring each side and expanding.

LEMMA 13. Let $x$ and $y$ be nonzero elements in an inner product space. If $m \geqq 0, m$ an integer, then

$$
\left|\frac{x}{|x|^{m+2}}-\frac{y}{|y|^{m+2}}\right| \leqq|x-y| \frac{p_{m}(x, y)}{|x|^{m+1}|y|^{m+1}}
$$

where $p_{m}(x, y)=\sum_{k=0}^{m}|x|^{m-k}|y|^{k}$. Equality holds only if $y$ is a positive multiple of $x$.

Proof. If $|x|=|y|$, the left side of (5.5) becomes $|x|^{-m-2}|x-y|$, and the right side becomes $(m+1)|x|^{-m-2}|x-y|$. If $|x| \neq|y|$, we apply (5.2), observing that

$$
p_{m}(x, y)=\left(|x|^{m+1}-|y|^{m+1}\right) /(|x|-|y|) .
$$

Proof of Theorem 2. Applying elementary estimates to (2.2), we obtain

$$
|T g(x)| \leqq M(n)\|g\|_{-\sigma}\left[I_{1}(x)+I_{2}(x)\right]
$$

where

$$
\begin{aligned}
& I_{1}(x)=\int_{|y| \geqq 1}|x-y|^{1-n} d y \\
& I_{2}(x)=\int_{|y| \geqq 1}|x-y|^{1-n}|y|^{-\sigma} d y .
\end{aligned}
$$


It is well known ([6], p. 53) that since the region of integration is bounded, $I_{1}(x)$ is uniformly bounded for $x$ in $R^{n}$ by a constant depending on $n$, and in particular we have

$$
I_{1}(x) \leqq M(n) \text { for }|x| \leqq 2 .
$$

If $|x| \geqq 2$, then in $I_{1}$ we have $|x-y| \geqq|x| / 2$, and

(5.9) $\quad I_{1}(x) \leqq \int_{|y| \leqq 1}(|x| / 2)^{1-n} d y \leqq M(n)|x|^{1-n}$ for $|x| \geqq 2$.

If $|x| \leqq 2$, then

$$
\begin{aligned}
I_{2}(x) & \leqq \int_{1 \leqq|y| \leqq 4}|x-y|^{1-n} d y+\int_{|y| \geqq 4}(|y| / 2)^{1-n}|y|^{-\sigma} d y \\
& \leqq M(n)+M(n) 4^{1-\sigma} /(\sigma-1)
\end{aligned}
$$

(again we used the result in [6], p. 53). Thus

$$
I_{2}(x) \leqq M(n, \sigma) \text { for }|x| \leqq 2 .
$$

Using another well-known result result for integrals of this type ([6], p. 200), we have

$$
I_{2}(x) \leqq \int_{R^{n}}|x-y|^{1-n}|y|^{-\sigma} d y \leqq M(n, \sigma)|x|^{1-\sigma} \quad \text { for } \quad x \neq 0
$$

Combining (5.6)-(5.12), we obtain (2.7). The proof of (2.8) is the same, since (5.6) holds with $T$ and $g$ replaced by $R$ and $u$.

To prove the Hölder continuity of $T g$, we use (2.2) and (5.5) to derive, for $|x|,|z| \leqq r, r \geqq 1$,

$$
\begin{aligned}
& |\operatorname{Tg}(x)-\operatorname{Tg}(z)| \\
& \quad \leqq M(n) \int_{R^{n}}|x-z|\left(\sum_{k=1}^{n-1}|x-y|^{-k}|z-y|^{-n+k}|g(y)|\right) d y \\
& \quad \leqq|x-z|\|g\|_{-\sigma} M(n) \sum_{k=1}^{n-1}\left(J_{k}+L_{k}+Q_{k}\right)
\end{aligned}
$$

where

$$
J_{k}=\int_{|y-x| \leqq 2|z-x|}|y-x|^{-k}|y-z|^{-n+k} d y
$$




$$
\begin{aligned}
L_{k} & =\int_{2|z-x| \leqq|y-x| \leqq 4 r}|y-x|^{-k}|y-z|^{-n+k} d y \\
Q_{k} & =\int_{|y| \geqq 3 r}|y-x|^{-k}|y-z|^{-n+k}|y|^{-\sigma} d y .
\end{aligned}
$$

In $J_{k}$ we make the change of variable $\xi=(y-x) /|z-x|$ and obtain

$$
J_{k}=\int_{|\xi| \leqq 2}|\xi|^{-k}|\xi-(z-x) /| z-x||^{-n+k} d \xi \leqq M(n) .
$$

(Note that by symmetry $J_{k}$ has the same value for all $z$ and $x$.) In $L_{k}$ we have $|y-z| \geqq|y-x| / 2$, and

$$
\begin{aligned}
L_{k} & \leqq 2^{n-k} \int_{2|z-x| \leqq|y-x| \leqq 4 r}|y-x|^{-n} d y \\
& \leqq M(n)(\log 4 r-\log 2|z-x|) \\
& \leqq M(r, n)+M(n)|\log | x-z||
\end{aligned}
$$

In $Q_{k}$ we have $|y-x| \geqq|y| / 2,|y-z| \geqq|y| / 2$, and

(5.16) $Q_{k} \leqq 2^{n} \int_{|y| \geqq 3 r}|y|^{-n-\sigma} d y \leqq M(n) \frac{(3 r)^{-\sigma}}{\sigma} \leqq \frac{M(n)}{3 r}=M(r, n)$.

Combining (5.14)-(5.16) with (5.12), we obtain the inequality

$$
|T g(x)-T g(z)| \leqq|x-z|\|g\|_{-\sigma}[M(r, n)+M(n)|\log | x-z||]
$$

which holds for fixed $r \geqq 1$ with $|x|,|z| \leqq r$. A similar inequality holds with $T$ and $g$ replaced by $R$ and $u$, and the statement on local Hölder continuity follows.

Proof of Theorem 3. We first consider the case $\sigma-n<\rho<$ $\sigma-1$. If $u \in B_{\rho}(n)$ then $b \cdot u \in B_{\rho-\sigma}$, and since $1<\sigma-\rho<n, \rho>$ $(1+\rho-\sigma)$, we have by Theorem 2 that $P u \in B_{1+\rho-\sigma}(n)$ and

$$
\begin{aligned}
\|P u\|_{\rho} & \leqq\|P u\|_{1+\rho-\sigma} \leqq M(n, \sigma-\rho)\|b \cdot u\|_{\rho-\sigma} \\
& \leqq M(n, \sigma-\rho)\|b\|_{-\sigma}\|u\|_{\rho} .
\end{aligned}
$$

Let $\left\{u_{m}\right\}_{m=1}^{\infty}$ be a bounded sequence in $B_{\rho}(n)$, say $\left\|u_{m}\right\|_{\rho} \leqq K$ for each $m$. By (5.18), 


$$
\left\|P u_{m}\right\|_{\rho} \leqq\left\|P u_{m}\right\|_{1+\rho-\sigma} \leqq M(n, \sigma-\rho)\|b\|_{-\sigma} K
$$

By (5.17), for fixed $r \geqq 1$ and $|x|,|z| \leqq r$,

$$
\begin{aligned}
& \left|P u_{m}(x)-P u_{m}(z)\right| \\
& \quad \leqq|x-z|\|b\|_{-\sigma} K[M(m, n)+M(n)|\log | x-z||]
\end{aligned}
$$

Thus the sequence $\left\{P u_{m}\right\}_{m=1}^{\infty}$ is uniformly bounded and equicontinuous on compact subsets of $R^{n}$. We use the Arzela-Ascoli theorem, while observing the behavior at infinity of these functions as determined by (5.19), to conclude that this sequence has a subsequence converging in the norm of $B_{\rho}(n)$.

Next consider $1-n<\rho \leqq \sigma-n$. Choose $\epsilon, 0<\epsilon<1$, such that $1+\epsilon-n<\rho$. If $u \in B_{\rho}(n)$ then $b \cdot u \in B_{-n+\epsilon}$ since $\rho-\sigma \leqq-n<$ $-n+\epsilon$. By Theorem 2, $P u \in B_{1+\epsilon-n}$, and

$$
\begin{aligned}
\|P u\|_{\rho} & \leqq\|P u\|_{1+\epsilon-n} \leqq M(n, n-\epsilon)\|b \cdot u\|_{\epsilon-n} \\
& \leqq M(n, n-\epsilon)\|b \cdot u\|_{\rho-\sigma} \leqq M(n, n-\epsilon)\|b\|_{-\sigma}\|u\|_{\rho} .
\end{aligned}
$$

Proceeding as in the previous case, the analogue of (5.19) is

$$
\left\|P u_{m}\right\|_{\rho} \leqq\left\|P u_{m}\right\|_{1+\epsilon-n} \leqq M(n, n-\epsilon)\|b\|_{-\sigma} K
$$

while (5.20) remains the same (after using \|\|$_{\epsilon-n} \leqq\|\|_{\rho-\sigma}$ ). The remainder of the proof is the same as in the previous case.

\section{REFERENCES}

1. L. Bers, Theory of Pseudo-analytic Functions, N. Y. U. Lecture Notes, 1953.

2. R. Courant and D. Hilbert, Methods of Mathematical Physics, Vol. 2, Interscience, New York, 1966.

3. A. Friedman, Bounded entire solutions of elliptic equations, Pacific J. Math., 44 (1973), 497-507.

4. D. Gilbarg and J. Serrin, On isolated singularities of solutions of second order elliptic differential equations, J. D'Analyse Mathématique, 4 (1954-6), 309-340.

5. R. P. Gilbert and G. Hile, Generalized hypercomplex function theory, Trans. Amer. Math. Soc., 195 (1974), 1-29.

6. G. Hellwig, Differential Operators of Mathematical Physics, Addison-Wesley, 1967.

7. J. Serrin, Entire solutions of nonlinear Poisson equations, Proc. London Math. Soc., 24 (1972), 348-366.

8. I. N. Vekua, Generalized Analytic Functions, Addison-Wesley, Reading, 1962.

Received July 16, 1975. This research was supported in part by National Science Foundation grant GP-20095. 



\section{Pacific Journal of Mathematics}

\section{Vol. 62, No. $1 \quad$ January, 1976}

Mieczyslaw Altman, Contractor directions, directional contractors and

directional contractions for solving equations . .................. 1

Michael Peter Anderson, Subgroups of finite index in profinite groups .........

Zvi Arad, Abelian and nilpotent subgroups of maximal order of groups of odd order

John David Baildon and Ruth Silverman, On starshaped sets and Helly-type theorems ..........................................

John W. Baker and R. C. Lacher, Some mappings which do not admit an

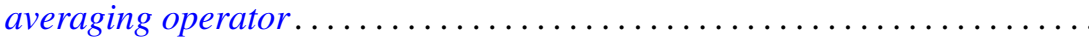

Joseph Barback, Composite numbers and prime regressive isols . . . . . . . . . .

David M. Boyd, Composition operators on $H^{p}(A) \ldots \ldots \ldots \ldots \ldots \ldots \ldots$

Maurice Chacron, Co-radical extension of PI rings . . . . . . . . . . . . .

Fred D. Crary, Some new engulfing theorems . . . . . . . . . . . . . . .

Victor Dannon and Dany Leviatan, A representation theorem for convolution transform with determining function in $L^{p} \ldots \ldots \ldots \ldots \ldots \ldots \ldots \ldots \ldots \ldots \ldots \ldots \ldots \ldots$

Mahlon M. Day, Lumpy subsets in left-amenable locally compact

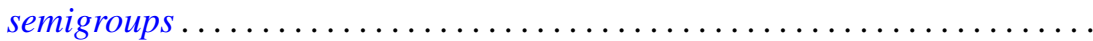

Michael A. Gauger, Some remarks on the center of the universal enveloping algebra of a classical simple Lie algebra . .

David K. Haley, Equational compactness and compact topologies in rings

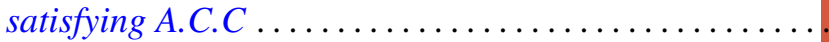

Raymond Heitmann, Generating ideals in Prüfer domains .

Gerald Norman Hile, Entire solutions of linear elliptic equations with

Laplacian principal part. .

Richard Oscar Hill, Moore-Postnikov towers for fibrations in which $\pi_{1}$ (fiber) is non-abelian

John Rast Hubbard, Approximation of compact homogeneous maps . .

Russell L. Merris, Relations among generalized matrix functions . .

V. S. Ramamurthi and Edgar Andrews Rutter, On cotorsion radicals ...

Ralph Tyrrell Rockafellar and Roger Jean-Baptiste Robert Wets, Stochastic

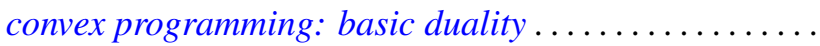

Alban J. Roques, Local evolution systems in general Banach spaces ..

I. Bert Russak, An indirect sufficiency proof for problems with bounded state variables.

Richard Alexander Sanerib, Jr., Ultrafilters and the basis property. .

H. A. Seid, The decomposition of multiplication operators on $L_{p}$-spaces . .

Franklin D. Tall, The density topology .................. 